\title{
Knockdown of flotillin-2 inhibits lung surfactant secretion by alveolar type II cells
}

\author{
Narendranath Reddy Chintagari ${ }^{1}$, Deming Gou ${ }^{1}$, Lin Liu ${ }^{1}$ \\ ${ }^{I}$ Department of Physiological Sciences, Oklahoma State University, 264 McElroy Hall, Stillwater, OK 74078, USA \\ Cell Research (2008) 18:701-703. doi: 10.1038/cr.2008.55; published online 6 May 2008
}

\section{Dear Editor,}

Lung surfactant is stored in lamellar bodies and exocytosed following fusion of the lamellar bodies with the plasma membrane of alveolar type II (AT2) cells [1]. A number of proteins have been shown to be involved in surfactant secretion including SNAREs, NSF, $\alpha$-SNAP and annexin A2 [2, 3]. Lipid rafts enriched in SNAREs are crucial for surfactant secretion [4].

Flotillins are lipid raft marker proteins. There are two isoforms, flotillin-1 and flotillin-2. Both are expressed in many cell types including AT2 cells [4]. Several functions of flotillins have been reported including interaction with protein kinases and the cytoskeleton; formation of filopodia; insulin signaling; axonal regeneration and cell-cell contacts and membrane trafficking $[5,6]$. The evidence for a role of flotillins in exocytosis is lacking except for a recent study which indicated the participation of flotillin-1 in mast cell exocytosis [7].

We investigated the functional role of flotillins in surfactant secretion by decreasing the expression of flotillin- 1 and -2 using RNA interference. We used an adenoviral vector to express shRNAs because of its high transduction efficiency in primary cells. This vector contained a shRNA sequence under the control of the mouse U6 promoter with EGFP expression under the control of the CMV promoter (Supplementary information, Materials and Methods). We chose 3 siRNA sequences targeted to different regions for each isoform: 118-137, 522-540 and 584-603 of flotillin1 [F-1 shRNA (A), (B) and (C)] and 53-73, 602-620 and 1163-1182 of flotillin-2 [F-2 shRNA(A), (B) and (C)]. The construct with irrelevant sequences served as a virus control (VC). The cells which were not transduced with any virus

Correspondence: Lin Liu

Tel: 00-1-405-744-4526; Fax: 00-1-405-744-8263

E-mail: lin.liu@okstate.edu were used as blank controls (BC).

We cultured AT2 cells with an air-liquid culture system to maintain their phenotype and transduced them with adenoviruses for 5 days. F-1 shRNA (A) almost completely silenced flotillin-1 expression (Figure 1A). There was also a decrease in flotillin-2 expression although a significant amount of flotillin-2 remained in the F-1 shRNA(A)-treated AT2 cells (Figure 1B). Among F-2 shRNAs, F-2 shRNA(C) was the most effective in silencing flotillin-2 expression. This shRNA also reduced flotillin-1 expression (Figure $1 \mathrm{~A}$ and $1 \mathrm{~B})$, probably because the stability of flotillin- 1 is dependent on flotillin-2 [8]. Using F-1 shRNA (A) and F-2 shRNA (C), we determined the effect of flotillin-1 and -2 silencing on surfactant secretion. There were no statistically significant differences in the basal and stimulated secretions between control and F-1 shRNA (A) - treated cells (Figure 1C). However, F-2 shRNA (C) reduced stimulated surfactant secretion. The basal secretion appears to be decreased in the F-2 shRNA (C)-treated cells but the difference did not reach a significant level. The control virus had no effect on surfactant secretion.

We next examined whether the loss of flotillin affects lipid raft formation. Because large amounts of cells were needed for this experiment, we used L2 cells, a rat lung epithelial cell line that expresses flotillin-1 and -2 [4]. We transduced L2 cells with F-1 shRNA (A) or F-2 shRNA (C). Infection efficiencies increased with the multiplicity of infection (MOI) of the virus as revealed by EGFP fluorescence (Supplementary information, Figure S1A). Western blot analysis indicated a significant decrease in protein expression of flotillin-1 or -2 in a dose-dependent manner (Supplementary information, Figure S1B). When compared with blank and virus controls, there were no obvious differences in the amounts of protein and cholesterol of the raft fractions between control and flotillin-1 or -2 knockdown cells (Supplementary information, Figure S1C and S1D). When cholesterol concentration was expressed 
A
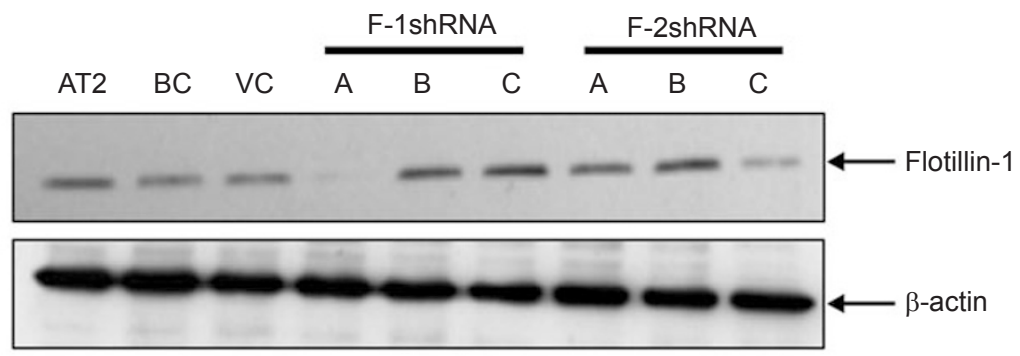

B
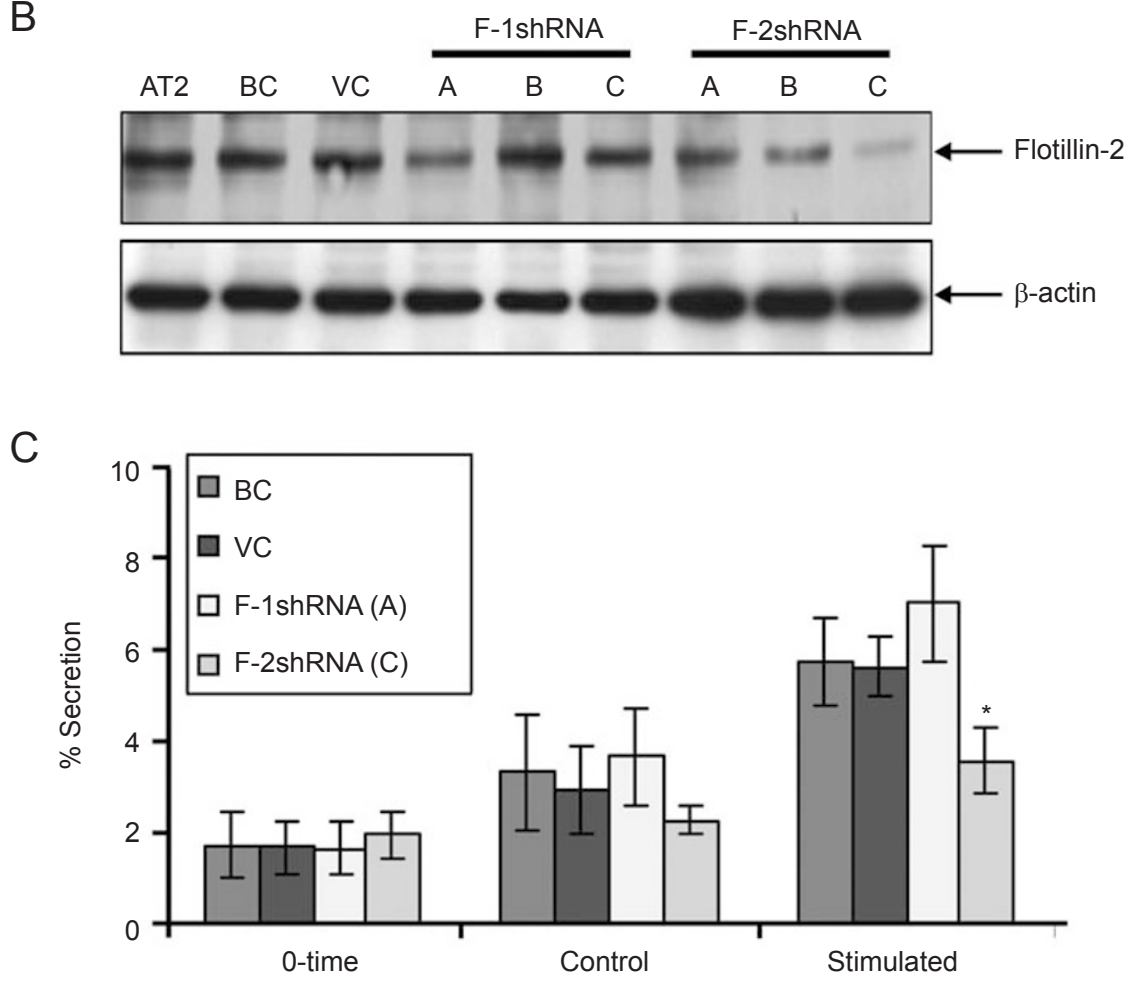

Figure 1 Effect of flotillin silencing on surfactant secretion. Alveolar type II (AT2) cells were transduced with different shRNA adenoviruses at an equal dose of 100 multiplicity of infection (MOI) and cultured for 5 days. Equal amounts of proteins were immunoblotted for flotillin-1 (A) and flotillin-2 (B). The same membranes were re-probed for $\beta$-actin to confirm equal loading. (C) Surfactant secretion. The control and flotillin knockdown cells were washed and equilibrated 30 min in serum-free medium (0-time). The cells were incubated $2.5 \mathrm{~h}$ in the absence (control) or presence of $100 \mu \mathrm{M} \mathrm{ATP}, 0.1 \mu \mathrm{M}$ phorbol 12-myristate 13acetate (PMA) and $10 \mu \mathrm{M}$ terbutaline (stimulated). The results were expressed as means \pm SE. ${ }^{*} P<0.05$ versus BC $(n \geq 3$ independent cell preparations, each assay in duplicates). BC: blank control, VC: virus control.

as $\mu \mathrm{g}$ per $\mu \mathrm{g}$ protein, cholesterol content in the lipid raft fraction was enriched 3-, 10-, and 20- fold in comparison with non-raft fractions 3,4 , and 5 .

SNARE proteins were enriched in lipid rafts of AT2 cells [4]. We examined whether knockdown of flotillin leads to dissociation of SNAREs with lipid rafts. Following silencing, little flotillin- 1 and -2 proteins were detected in any of the fractions, indicating an effective silencing. When raft fractions were probed for two plasma membrane SNARE proteins, syntaxin-2 and SNAP-23 [2] following silencing, there was no change in the association of these proteins with lipid rafts (Supplementary information, Figure S1E and S1F).

Lipid rafts are also reported to be involved in cholesterol homeostasis. We further investigated the effects of flotillin-1 and -2 silencing on cholesterol levels in L2 cells. Following silencing of flotillin- 1 and -2 , cell lysates were analyzed for total cholesterol levels. Our results indicated 
that there were no statistical significant differences in cholesterol levels under silencing conditions when compared with controls (Supplementary information, Figure S1G).

We conclude that flotillin-2 is required for surfactant secretion but not via influencing the association of SNAREs with the rafts or cholesterol homeostasis. The mechanisms by which flotillin-2 regulates surfactant secretion remain to be determined, but could be due to its effects on signaling pathways or cytosketetal rearrangement because flotillin-2 knockdown blocks the G-protein coupled receptor (GPCR)mediated activation of p38 MAPK [9] and trans-negative mutant flotillin-2 leads to faulty localization of Vav, resulting in altered cytoskeletal rearrangements [10].

\section{Acknowledgments}

We thank our colleague Dr Heidi Stricker for editorial assistance. This work was supported by NIH grants R01 HL-052146, R01 HL-071628 and R01 HL-083188 (LL).

\section{References}

1 Andreeva AV, Kutuzov MA, Voyno-Yasenetskaya TA. Regulation of Surfactant Secretion in Alveolar Type II cells. Am J Physiol Lung Cell Mol Physiol 2007; 293:L259-L271.

2 Abonyo BO, Gou D, Wang P, Narasaraju T, Wang Z, Liu L.
Syntaxin 2 and SNAP-23 are required for regulated surfactant secretion. Biochemistry 2004; 43:3499-3506.

3 Wang P, Chintagari NR, Gou D, Su L, Liu L. Physical and Functional Interactions of SNAP-23 with Annexin A2. Am J Respir Cell Mol Biol 2007; 37:467-476.

4 Chintagari NR, Jin N, Wang P, Narasaraju TA, Chen J, Liu L. Effect of cholesterol depletion on exocytosis of alveolar type II cells. Am J Respir Cell Mol Biol 2006; 34:677-687.

5 Glebov OO, Bright NA, Nichols BJ. Flotillin-1 defines a clathrinindependent endocytic pathway in mammalian cells. Nat Cell Biol 2006; 8:46-54.

6 Babuke T, Tikkanen R. Dissecting the molecular function of reggie/flotillin proteins. Eur J Cell Biol 2007; 86:525-532.

7 Kato N, Nakanishi M, Hirashima N. Flotillin-1 regulates IgE receptor-mediated signaling in rat basophilic leukemia (RBL2H3) cells. J Immunol 2006; 177:147-154.

8 Hoehne M, de Couet HG, Stuermer CA, Fischbach KF. Loss- and gain-of-function analysis of the lipid raft proteins Reggie/Flotillin in Drosophila: they are posttranslationally regulated, and misexpression interferes with wing and eye development. Mol Cell Neurosci 2005; 30:326-338.

9 Sugawara Y, Nishii H, Takahashi T, et al. The lipid raft proteins flotillins/reggies interact with Galphaq and are involved in Gqmediated $\mathrm{p} 38$ mitogen-activated protein kinase activation through tyrosine kinase. Cell Signal 2007; 19:1301-1308.

10 Langhorst MF, Reuter A, Luxenhofer G, et al. Preformed reggie/flotillin caps: stable priming platforms for macrodomain assembly in T cells. FASEB J 2006; 20:711-713.

(Supplementary Information is linked to the online version of the paper on the Cell Research website.) 